\title{
Sidewall friction in confined surface flows of granular materials
}

\author{
Patrick Richard ${ }^{1, *}$, Alexandre Valance ${ }^{2, * *}$, and Renaud Delannay ${ }^{2, * * *}$ \\ ${ }^{1}$ MAST-GPEM, Univ Gustave Eiffel, IFSTTAR, F-44344 Bouguenais, France \\ ${ }^{2}$ IPR, Université de Rennes 1, CNRS UMR 6251, Campus Beaulieu, F-35042 Rennes, France
}

\begin{abstract}
We report numerical simulations of surface granular flows confined between two sidewalls. These systems exhibit both very slow and very energetic flows. Zhu et al. [1] have shown that in energetic confined systems, the Froude number at sidewalls and the sidewall effective friction coefficient are linked through a unique relation. We show that this relation is also valid for creep flows. It is independent of the angle of the flow but depends on the sidewall-grain friction coefficient. Our results shed light on boundary conditions that have to be used at sidewalls in continuum theories aiming to capture the behavior of granular systems from creeping to energetic flows.
\end{abstract}

\section{Introduction}

Granular flows confined between two sidewalls are an interesting benchmark to study granular rheology for several reasons. To mention one of them, the presence of sidewalls may induce cooperative effects with a characteristic length much greater than the grain size [2]. Also, steady and fully developed (SFD) flows can be achieved at very important flow angles [3-5], the base friction supplemented by the sidewall friction being able to balance the driving component of the weight. Such types of flows have been recently studied by Brodu et al. [6] by means of discrete element method simulations. These authors have indeed shown that flows can be SFD at high angles and their results revealed the existence of new flow regimes characterized by (i) complex internal structures, (ii) secondary flows and (iii) heterogeneous particle volume fraction [6,7]. Recently Zhu et al. [1] have revisited these types of flows and obtained the following key result: the effective sidewall friction can be described as a unique function of a dimensionless number which is the analog of a Froude number. This result is interesting to establish boundary conditions for granular flows. Yet, the study of Zhu et al., [1] focused on energetic flows so the validity of the aforementioned relation to slow and creeping granular flows remains unanswered and deserves attention.

Here, by means of Discrete Element Method (DEM) simulations, we investigate the validity of Zhu's relation between Froude number and sidewall friction for a large range of the former quantity i.e. from creeping to energetic flows. For that purpose we carry out numerical simulations of confined surface in the so-called sidewall stabilized heap (SSH) flow geometry for which, creeping, flowing and gazeous zones can be observed simultaneously.

*e-mail: patrick.richard@univ-eiffel.fr

**e-mail: alexandre.valance@univ-rennes1.fr

***e-mail: renaud.delannay@univ-rennes1.fr

A video is available at https://doi.org/10.48448/wzk3-ky80
The outline of the paper is the following. First, we will present both the geometry of the system and, briefly, the numerical method (section 2). Section 3 will be devoted to the study of the key parameters of the system at sidewall: streamwise velocity and effective friction coefficient. In section 4 we will probe the validity of Zhu's relation for $\mathrm{SSH}$ flows i.e. for small and large values of the Froude number. Finally we will present our conclusions.

\section{Geometry and Methods}

As mentioned in the introduction, the geometry used in this study aims to model so-called sidewall stabilized heaps (SSH) initially introduced experimentally in [8]. They consist of a surface flow on a heap between two flat and frictional sidewalls and driven by gravity. This surface flow occurs above a quasistatic heap stabilized by sidewall friction. Experimentally the flow angle increases with the input flow rate and can be much steeper than the angles for which a SFD flow can be achieved in an unconfined flow geometry. Another important remark should be mentioned here. These flows are influenced by the confinement even at very large widths between sidewalls [9]. To simulate numerically SSH flows we use an inclined $3 D$ cell (see Fig. 1) similar to those used in [10-12]. The angle between the horizontal and the main flow direction ( $x$-direction) is called $\theta$. In such types of geometries, the angle of the flow and the flow rate are related as long as there are enough grains in the system to ensure the presence of a creep zone above which a flow occurs [13]. Note that, in simulations, the angle is chosen by the user and the system adapts its flow rate. In contrast, in experiments, the user choose the input flow rate (which in case of SFD flows is equal to the output flow rate) and the system adapts the flow angle consequently. The size of the cell in the $x$ - direction is set to $L_{X} \approx 25 d$ with periodic boundary conditions in this direction. In the 


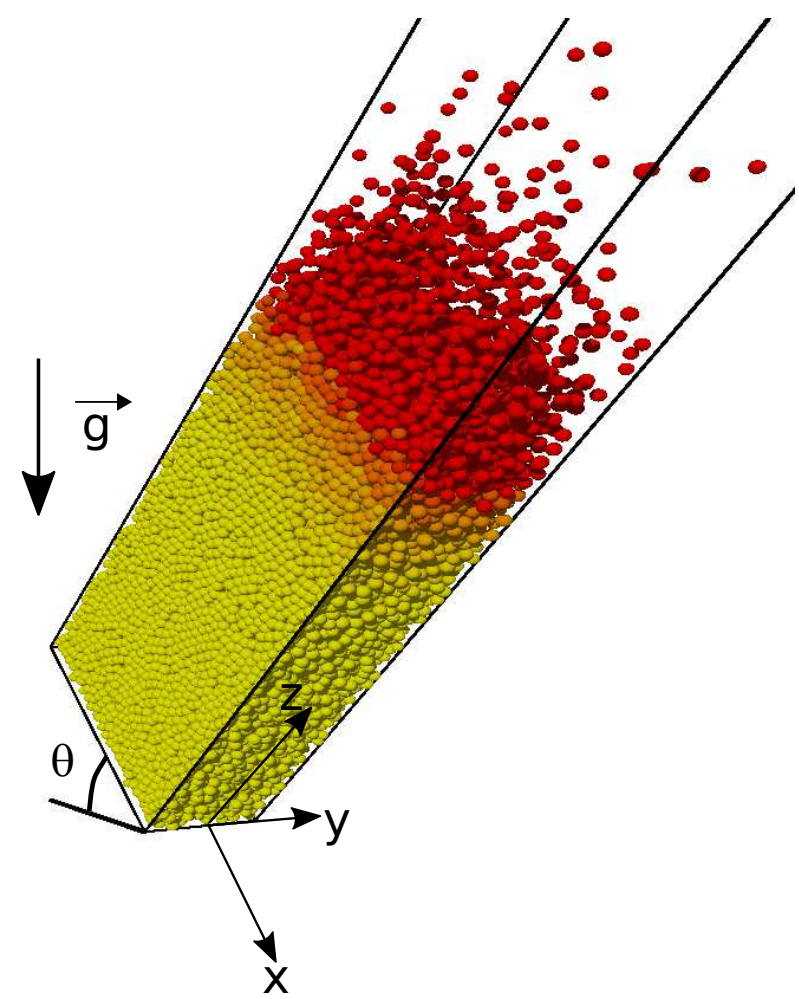

Figure 1. Typical snapshot for SSH flows: $W / d=10$ and $N=$ 15,000. The angle of the flow is $\theta=45^{\circ}$, the coefficient of restitution $e_{n}$ is equal to 0.88 , and the grain-grain and grainssidewall friction coefficients (respectively $f_{g}$ and $f_{w}$ ) are set to 0.5 . Flow is directed down the incline along the $x$-axis. Two sidewalls confining the system are parallel to the $(x z)$ plane.

$z$-direction (i.e. normal to the free surface of the flow) the size of the cell is set to large values and thus considered as infinite. In the $y$-direction, the flow is confined by two flat frictional sidewalls located at $y=-W / 2$ and $y=W / 2$ with $W=10 d$. The bottom of the cell, located at $z=0$, is made bumpy by pouring under gravity $\mathbf{g}$ a large number of grains in the cell and by gluing those that are in contact with the plane $z=0$ and removing the others.

We use soft-sphere molecular dynamics simulations developed internally [10] for which $N=15,000$ may overlap slightly. The normal force between grains, $F_{n}$, is classically modelled by a spring and a dashpot: $F_{n}=k_{n} \delta-\gamma_{n} \dot{\delta}$ where $k_{n}$ and $\gamma_{n}$ are respectively the stiffness of the spring and the viscosity of the dashpot, $\delta$ the overlap between grains and $\dot{\delta}$ its derivative with respect to time. The stiffness is set to $5.6 \times 10^{6} \mathrm{mg} / \mathrm{d}$ and $\gamma_{n}$ is chosen such as the normal restitution coefficient is equal to 0.88 [13]. The tangential force is modelled by a spring, $F_{t}=k_{t} u_{t}$, where $k_{t}=2 k_{n} / 7$. Its deformation $u_{t}$ (i.e. the elastic tangential displacement between grains) is bounded to satisfy Coulomb law $F_{t}=\mu F_{n}$, where $\mu$ is the friction coefficient which, in the remainder of the paper, is set to $\mu=f_{g}=0.5$ for a grain-grain contact. The walls are treated like spheres of infinite mass and radius. The normal restitution coefficient of the grain-wall interaction is the same than that used for the grain-grain interactions. In contrast the value of the friction coefficient between the grains and the walls, $f_{w}$, will be varied to study its effect. To avoid any structural ordering the diameter of the grains is uniformly distributed between $0.8\langle d\rangle$ and $1.2\langle d\rangle$ where $\langle d\rangle$ is the average grain diameter. Initially, the kinetic energy of the system is set to an important value [13] such as the SFD state obtained after a transient does not show any sign of the initial structure. More details on the the simulation procedure can be found in $[13,14]$.

\section{Velocity and effective friction at sidewalls}

To characterize the flow we first focus on the streamwise velocity of the particle at sidewalls, $V_{x, w}$, and report its vertical profile on Fig. 2. Note also that this quantity will be used latter (Sect. 4) to determine the Froude number. In agreement with the literature, the system displays a wide range of behaviors from creep in the vicinity of the bottom, characterized by an exponential variation of the velocity (see inset of Fig. 2), to very energetic flows in the vicinity of the free surface. In [14] we have shown that in such systems four zones can be defined from the velocity profile (from bottom to free surface): (i) a creeping zone, (ii) a buffer zone, (iii) a flow zone and (iv) a gazeous zone. It should be pointed out that the volume fraction (not shown here) varies from $\approx 0.6$ close to the bottom to 0 at the free surface and that this variation, in contrast to that observed for unconfined flows, is very smooth. A full study of the characteristic length of this variation can be founded in [13]. As mentioned in the introduction and in

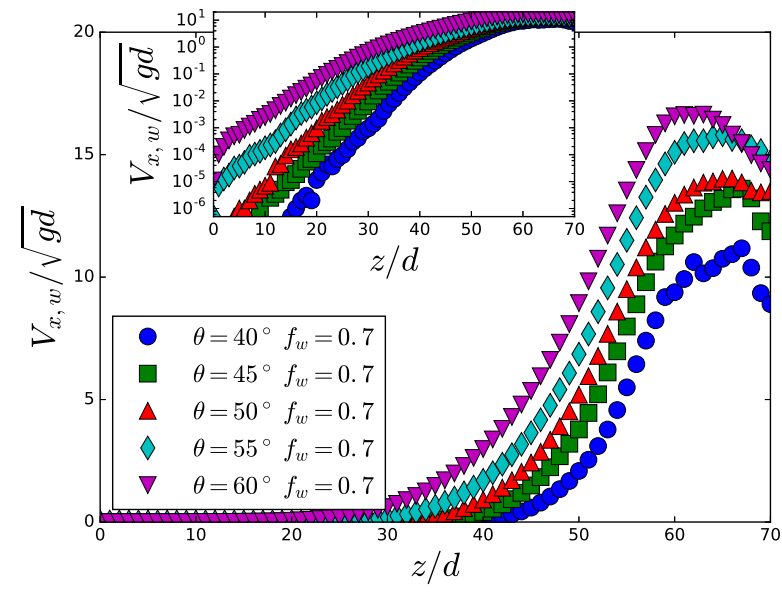

Figure 2. The velocity in the streamwise direction and at sidewalls, $V_{x, w}$ increases with flow angle and displays an exponential variation characteristic of creeping in the vicinity of the bottom (inset).

section 3, we have a system for which the range of variation of the grains' velocity is huge and so is the range of Froude numbers. Thus those systems are indeed an interesting benchmark to verify if Zhu's relation holds for small Froude numbers. It should also be pointed out that, obviously, the angle of the flow has an influence on the 
sidewall velocity, the latter being found to increase with the former. Yet the shape of the profiles is similar [13, 14]. To understand confined granular flows and more precisely how they interact with the confining sidewalls, a key observable is the effective sidewall friction coefficient. It is defined as $\mu_{\tau} \equiv\left\|\tau_{w}\right\| /\left\|\sigma_{y y}^{w}\right\|$, where $\sigma_{\alpha \beta}^{w}$ is the stress at sidewalls and $\tau_{w} \equiv \sigma_{y x}^{w} \mathbf{e}_{\mathbf{x}}+\sigma_{y z}^{w} \mathbf{e}_{\mathbf{z}}$ with $\mathbf{e}_{\mathbf{x}}$ and $\mathbf{e}_{\mathbf{z}}$ being unit vectors along the $x$ - and $z$-directions, respectively.

We have reported on Fig. 3 the vertical profiles of the sidewall friction for several flow angles but constant $f_{g}$ and $f_{w}$ (0.5 and 0.7 respectively). The effective friction co-

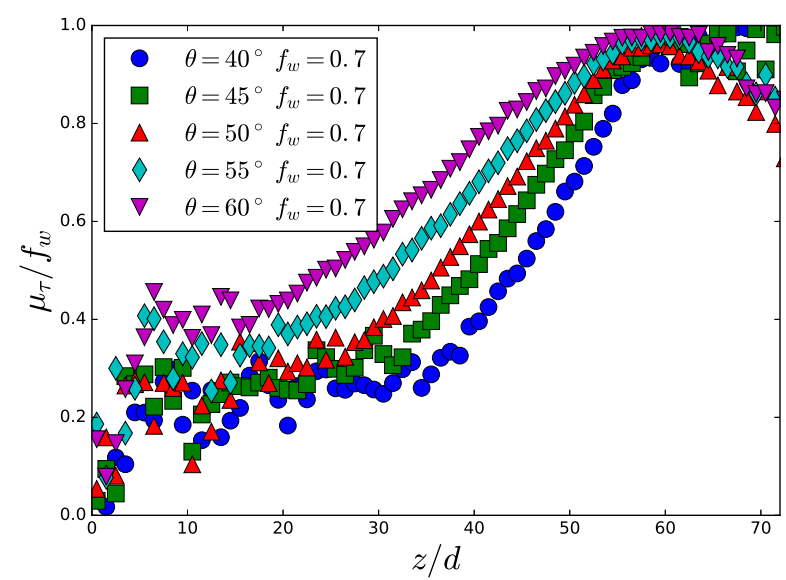

Figure 3. The effective friction coefficient on sidewalls, $\mu_{\tau}$, is strongly influenced by confinement. In the vicinity of the free surface, it is close to the grain-sidewall friction coefficient, $f_{w}$ Then, it weakens and, in the creep zone, reaches a constant value.

efficient, $\mu_{\tau}$, is found to be close to microscopic friction coefficient between sidewalls and grains in the flow zone, $f_{w}$ (Fig. 3). In contrast, in the creep zone, the Coulomb threshold is far from being reached and the corresponding ratios tangential force to normal force remains below $f_{w}$. This phenomenon is known as the friction weakening phenomenon $[2,10]$. The effective friction seems to tends towards a constant value deep in the creep zone and is equal to zero only at the bottom. The length required for the effective friction coefficient to weaken to the aforementioned constant value increases with the flow angle.

\section{Relation between effective sidewall friction and Froude number}

Following Zhu et al. [1], we have computed the quantity $F_{r}=V_{x, w} / \sqrt{\sigma_{y y}^{w} / \rho}$ where $\rho$ is the material density of the grains. This quantity can be seen as a Froude number in the vicinity of sidewalls. The flows being SFD, the Froude number is determined for each height within the flow. We first present in Fig. 4(a) the evolution of the ratio of the effective sidewall friction to the grain-sidewall friction coefficient, i.e. $\mu_{\tau} / f_{w}$, versus the Froude number for constant values of $f_{g}$ and $f_{w}$ and several angles. Remarkably, all the data collapse reasonably on a single master curve whereas, as seen previously in Fig. 3, the effective friction coefficient depends on the angle. We also report the same data
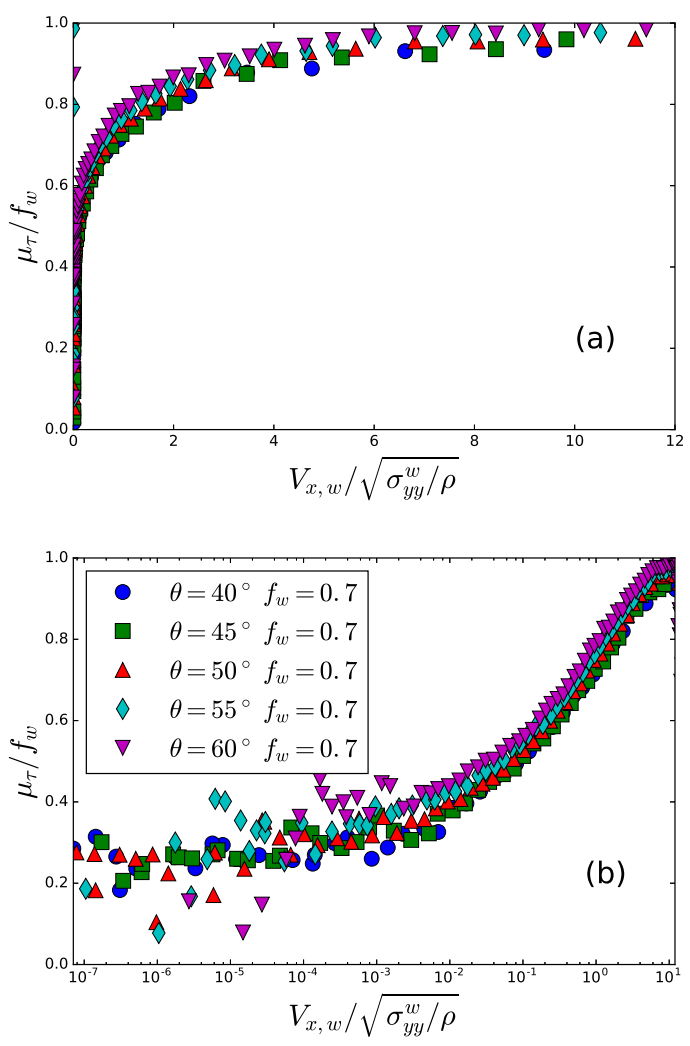

Figure 4. For a given system, the relation between the effective sidewall friction coefficient $\mu_{\tau}$ and the Froude number at sidewalls is unique (a). This is true even at small Froude numbers (b).

in loglin scale (Fig. 4(b)) to investigate more carefully the data at low Froude numbers. Three remarks deserve to be pointed out. First, these curves show that the relation between $\mu_{\tau}$ and the Froude number reported in [1] remains valid at low Froude number. Consequently it seems such Zhu's relation can indeed be used to derive boundary conditions for a large range of types of flows (including creeping flows), at least for flat and frictional sidewalls. Second, Zhu et al. [1] have propose an exponential functional form to model their data. For important Froude number the evolution of $\mu_{\tau}$ is indeed compatible with an exponential behavior. However, for small Froude number the exponential behavior is no more valid. It is thus tempting to define two regimes, one for large values of Froude number with an exponential functional form, and another one for small values. Third, it should be pointed out that the effective friction coefficient does not tend towards zero for small Froude number but seems to reach a constant value. This is consistent with the results reported in Sect. 3

To go deeper in our understanding, we have studied the evolution of the relationship between the effective friction coefficient and the Froude number with the grain-sidewall friction coefficient $f_{w}$. For that purpose we report on Fig. 5 $\mu_{\tau} / f_{w}$ versus the Froude number for a constant flow angle but several grain-sidewall friction coefficients. We found that the aforementioned relation depends on $f_{w}$. For a given Froude number, the effective friction coefficient in- 


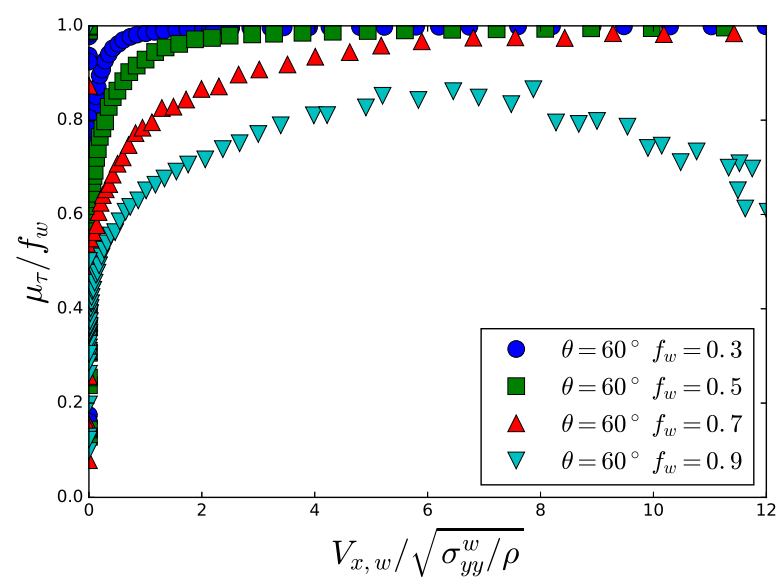

Figure 5. Zhu's relation (ratio of the effective friction coefficient at sidewalls to the microscopic grain-sidewall friction coefficient versus the Froude number at sidewalls $V_{x, w} / \sqrt{\sigma_{x x}^{w} / \rho}$ ) is found to depend on the grain-sidewall microscopic friction coefficient.

creases with increasing $f_{w}$. This means that Zhu's relation is unique for a given system but depends on the grain properties. Note that when $f_{w}$ tends towards zero, the functional form representing the evolution of $\mu_{\tau} / f_{w}$ versus the Froude number tends towards a step function. The next step of this study consists in finding a functional form for the latter relation valid both or large and small Froude numbers. Our result show that this functional has to depend on the grain and sidewall properties.

\section{Conclusion}

We have studied the properties of granular surface flows driven by gravity and confined between two flat but frictional sidewalls. In such systems a continuum is observed between quasistatic flow where creeping occurs and very energetic flows. We have shown that the unique relation established in [1] between the sidewall effective friction coefficient and the Froude number remains valid even at small Froude number. For a given system, this relation is unique. Yet it depends on the system properties (e.g. the microscopic friction coefficient between grains and sidewalls which is a parameter of the simulations). This results suggest that the Froude number should be used to derive boundary conditions at sidewalls, at least for flat and frictional sidewalls.

As mentioned above the next step of this study is to find a functional form able to fit the numerical data or both small and large values of Froude numbers and link the parameters of this form to observables. Also it should be interesting to investigate other confined geometries to strengthen the results presented here.

\section{References}

[1] Y. Zhu, R. Delannay, R. Valance, Gran. Matt. 22, 82 (2020)

[2] R. Artoni, P. Richard, Phys. Rev. Lett. 115, 158001 (2015)

[3] A.J. Holyoake, J.N. McElwaine, J. Fluid Mech. 710, 35 (2012)

[4] T. Börzsönyi, R.E. Ecke, J.N. McElwaine, Phys. Rev. Lett. 103, 178302 (2009)

[5] S. Zhang, G. Yang, P. Lin, L. Chen, L. Yang, Eur. Phys. J. E 42, 40 (2019)

[6] N. Brodu, R. Delannay, A. Valance, P. Richard, J. Fluid Mech. 769, 218 (2015)

[7] N. Brodu, P. Richard, R. Delannay, Phys. Rev. E 87, 022202 (2013)

[8] N. Taberlet, P. Richard, A. Valance, W. Losert, J.M. Pasini, J.T. Jenkins, R. Delannay, Phys. Rev. Lett. 91, 264301 (2003)

[9] P. Jop, Y. Forterre, O. Pouliquen, J. Fluid Mech. 541, 167 (2005)

[10] P. Richard, A. Valance, J.F. Métayer, P. Sanchez, J. Crassous, M. Louge, R. Delannay, Phys. Rev. Lett. 101, 248002 ( 4) (2008)

[11] D. Gollin, D. Berzi, E. Bowman, Gran. Matt. 19, 56 (2017)

[12] D. Berzi, J.T. Jenkins, P. Richard, Soft Matter 15, 7173 (2019)

[13] P. Richard, A. Valance, R. Delannay, P. Boltenhagen, in preparation (2021)

[14] P. Richard, R. Artoni, A. Valance, R. Delannay, Gran. Matt. 22, 81 (2020) 